Freilich sind diese Werthe nur annäherncle, da die angewendeten Verfahren nicht den Bedingungen einer vollständig genauen analytischen Methode entsprechen. Es besteht also das Haselnussöl aus neun Zehnteln des Oelsäureglycerides und einem Zehntel des Palmitins mit geringen Mengen des Stearins. Somit näherte sich die Angabe König's der Wahrheit am meisten, bis auf die Arachinsäure, welche im vorliegenden Falle nicht nachgewiesen werden konnte, während Schöttler die Stearinsäure nicht angeführt hat, obwohl sich diese, wenn auch in geringerer Menge, im Haselnussöle vorfindet.

\title{
Die relative Verdaulichkeit einiger Nahrungsfette im Darmkanal des Menschen.
}

\author{
II. Palmin.
}

Von

\author{
I). H. Lührig. \\ I. Assistent am chemischen Lintersuchungsamte der stadt Altona.
}

Das Bestreben, die beiden wichtigsten und in ihrer Verwendung verbreitetsten thierischen Nabrungsfette, Butter und Schweineschmalz, durch geeignete billige Kunstprodukte zu ersetzen, hat u. a. dazu geführt, die theilweise in ungeheuren Mengen in der Natur aufgespeicherten pflanzlichen Oele und Fette zur Fabrikation entsprechender Ersatzmittel heranzuziehen und sie in Folge dessen für die menschliche Ernährung nutzbar zu machen. Abgesehen von der seit Alter's her bekannten Verwendung gewisser mit einem specifischen Geschmacke behafteten Pflanzenöle (Olivenöl, Mohnöl, Rüböl etc.) zu den mannigfachsten Speisezwecken, verdienen neuerdings dic in grossen Mengen eingeführten Oele mit indifferentem Geschmack (Erdnuss-, Sesam-, Baumwollsamenöl etc.), die theils für sich allein zu Speisezwecken benutzt, theils mit thierischen Fetten gemischt zur Bereitung von Margarine und Kunstspeisefett vielfache Verwendung finden, in erhöhtem Maasse Beachtung. Bei der grossen Bedeutung der Fette für die menschliche Ernährung sind die Bestrebungen, an Stelle der theueren thierischen Fette, welche für die ärmere Bevölkerung gewissermaassen ein Luxusartikel geworden sind, gute und vor allem billige Ersatzmittel zu setzen, nicht nur von allgemeinen Gesichtspunkten aus zu rechtfertigen und zu billigen, sondern diesen Präparaten kommt, sofern sie obige beiden Eigenschaften besitzen, ausserdem eine erhebliche volkswirthschaftliche Bedeutung zu.

Bei diesen Bestrebungen, billige Nahrungsfette herzustellen, die einerseits eine möglichst vielseitige Nutzanwendung zulassen, andererseits bezüglich ihrer Herstellung allen an ein menschliches Nahrungsmittel zu stellenden hygienischen Anforderungen entsprechen, ausserdem im Aussehen und der Konsistenz bestehenden Gebräuchen Rechnung tragen und sich der menschlichen Geschmacksrichtung thunlichst anpassen, war es naheliegend, das in den Fruchtkernen 
der Kokosnuss reichlich aufgespeicherte Fett, im isolirten Zustande schlechthin „Kokosnussbutter" genannt, zum Ausgangsmaterial für die Herstellung eines rein pflanzlichen Nahrungsfettes zu machen. Die in Folge ihrer Anspruchslosigkeit vornehmlich in den Küstenländern der Tropen über grosse Flächen verbreitete Kokospalme (Cocos nucifera L.) zeichnet sich durch ausserordentliche Fruchtbarkeit aus, sodass die in enormen Mengen zur Verfügung stehenden Früchte ${ }^{1}$ ) an Ort und Stclle nur einen geringen Werth besitzen. Bei der Billigkeit des Rohstoffes war von vornherein anzunehmen, dass seine Verwendung zu den mannigfachsten Zwecken, auch zu Speisezwecken, angestrebt wurde.

Das aus den Fruchtkernen auf verschiedene Weise gewonnene rohe Kokosfett ist in diesem Zustande als Nahrungsfett noch nicht verwendbar, sondern muss erst einem Reinigungsprocess unterworfen werden, welcher die Entfernung gewisser, Geruch und Geschmack beeinflussenden Stoffe, besonders der freien Fettsäuren bezweckt.

Wenn es trotzdem keinem der seit dem letzten Jahrzehnt vielfach in den Verkehr gebrachten Präparate möglich war, sich ein dauerndes Absatzgebiet zu erhalten, so lag das unter anderem daran, dass es trotz Anwendung mancher als zweckmässig empfohlener Raffinationsverfahren nicht gelang, die Produkte, die schon nach verhältnissmässig kurzer Zeit Neigung zum Ranzigwerden zeigten, vor ihrer leichten Zersetzlichkeit zu schützen.

Die allen bisherigen Präparaten in mehr oder weniger starkem Grade anhaftendon Mängel in Bezug auf Haltbarkeit und leichte Zersetzlichkeit scheinen durch ein neues, allerdings geheim gehaltenes Verfahren zur Herstellung einer fast säurefreien Kokosnussbutter, die seit etwa Jahresfrist unter dem gesetzlich geschützten Namen "Palmin" in den Handel gebracht wird, im Wesentlichen gehoben zu sein. Das mit „Palmin" bezeichnete Erzeugniss ist von rein weisser Farbe, nahezu säure- und wasserfrei, ohne körnige Struktur und besitzt einen angenehmen, nussartigen Geruch und Geschmack. Auf die Zunge gebracht zerfliesst es leicht, dabei einen milden Geschmack hinterlassend. Die eigenartige Konsistenz, die durch die Härte des Fettes bedingt wird, lässt eine allgemeine Verwendung; wie etwa die Butter sie besitzt, nicht $z u$, sodass dieselbe in Folge dessen vornehmlich auf Back- und Küchenzwecke beschränkt bleibt.

Die Haltbarkeit der Waare lässt nichts $\mathrm{zu}$ wünschen übrig. Ich habe Palmin in Blechdosen bei Zimmertemperatur bis zu 8 Wochen aufbewahrt, ohne

1) Die reife, eiförmige, ctwas stumpf-dreikantige Frucht enthält den mit dem Endocarp verwachsenen Samen, dessen festes Nährgewebe, eine weisse ölreiche peripherisch gelagerte Masse, eine Dicke von 10-20 mm erreicht und unter dem Nannen "Copra“ als HIandelsartikel bekannt ist. Der in der IIöhlung dieses festen Theiles des Năhrgewebes in der Regel noch vorhandene flüssige Theil desselben, die "Kolkosmilch", welche bei der Keimung des Samens zuerst zur Verwendung gelangt, ist in den Tropen als ausserordentlich erfrischendes Getränk ron süss-räuterlichem Gesehmack sehr geschätzt. Die Zusammensetzung des festen Nihhrgewebes ist mach J. König's Chemie der menschlichen Nahrumgs- und Genussmittel folgende:

$\begin{array}{cccccc}\text { Wasser } & \text { Eiweiss } & \text { Fett } & \text { Kohlenhydrate } & \text { Rohfaser } & \text { Asche } \\ 5,81 \% & 8,88 \% & 62,00 \% & 12,44 \% & 4,06 \% & 1,81^{\circ} \% \text {. }\end{array}$


dass irgend welche wahınehmbaren Veränderungen im Geruch oder Geschmack zu erkennen gewesen wären; auch Aenderungen des Säuregrades konnte ich in genannter Zeit nicht feststellen. Bei noch längerer Aufbewahrung nehmen allerdings die unter Verwendung solch' älterer Präparate ${ }^{1}$ ) hergestellten Speisen, insbesondere beim Braten, einen schwach seifigen Geschmack an, was deren Gebrauch zu solchen Zwecken vou selbst aussehliesst.

Nach seinen chemischen Eigenschaften erweist sich Palmin als eine reine, fast säurefreie Kokosnussbutter, welche sich bekanntermaassen in ihrer Zusammensetzung von den meisten pflanzlichen Fetten durch den ungewöhnlich grossen Gehalt an Triglyceriden der Myristin- und Laurinsäure unterscheidet. Sie enthält ferner ausser Palmitin die Glyceride der Capron-, Capryl- und Caprinsäure.

Zur Identificirung dient einmal die verhältnissmässig hohe Verseifungszahl und niedrige Jodzahl, ferner die verhältnissmässig hohe Reichert-Meissl-Zahl, bedingt durch den Gehalt an niederen Fettsäuren. Mit Irülfe dieser sehr beständigen Werthe gelingt es leicht, festzustellen, ob eine reine Kokosbutter vorliegt oder ein Gemisch mit anderen Fetten. Dic für Palmin ermittelten chemischen Konstanten (Mittel aus mehreren Bestimmungen) sind im Folgenden wiedergegeben.

\begin{tabular}{l|c|c|c|c|c|c}
\hline & $\begin{array}{c}\text { Schmelz- } \\
\text { punkt }\end{array}$ & $\begin{array}{c}\text { Erstarrungs- } \\
\text { punkt }\end{array}$ & Jodzahl & $\begin{array}{c}\text { Verseifungs- } \\
\text { zahl }\end{array}$ & $\begin{array}{c}\text { Reichert- } \\
\text { Meissl- } \\
\text { Zahl }\end{array}$ & $\begin{array}{c}\text { Refraktion } \\
\text { bei } 40^{\circ} \mathrm{c} .\end{array}$ \\
\hline Palmin . . . . & $25^{0}$ & 22,4 & 7,27 & 260,7 & 7,85 & 35,1 \\
Cnlösl. Fettsäuren & $25-25,3^{\circ}$ & 22,8 & 6,90 & 271,1 & - & $\mathbf{1 8 , 1}$
\end{tabular}

Charakteristisch erweist sich Palmin beim Schmelzen und darauf folgenden Wiedererstarren. Bei $25^{\circ}$ schmilzt es zu einer klaren Flüssigkeit, welche bis tief unter den Schmelzpunkt tlüssig bleibt. Nachdem die $\Lambda$ bkiihlung bis 19,8 vorgeschritten ist, steigt das Thermometer schnell und stellt sich bis zum völligen Erstarren beständig bei 22,3 bis $22,4^{\circ}$ ein. Eine ganz ähnliche Erscheinung zeigen die unlöslichen Fettsäuren.

Vom hygienischen Standpunkt hat das Palmin im Vergleich zu den gebräuchlichen thierischen Nahrungsfetten und deren Surrogaten insofern einen Vorzug, als jedwede gesundheitlichen Bedenken fortfallen, die unter Umständen bei den letztgenannten Fetten, wenn sie von kranken Thiereu stammen, nicht von der Hand zu weisen sind.

Lm die Keimfreiheit festzustellen, wurden von verschiedenen Stellen der mir zur Verfügung stehenden und in Blechdosen zum Versand gelangenden zwei Proben Palmin mit sterilisirtem Messer Stücke von Haselnussgrösse herausgeschnitten, auf einen sterilen Platindraht gesteckt und mit der Flamme eines

1) Da es sich im rorliegeuden Fulle bei der Prüfung auf Haltbarkeit un verhälnissmässig kleine Proben (ca. $300 \mathrm{~g}$ ) handelte, die offen an der Iuft aufbewahrt wurden, vermag ich anf Grund des erwälnten Vexhaltens nicht ohne Weiteres zu folgern, ob bei grösseren Proben, die vor Luftzutritt möglichst gesehützt sind, sich derselbe oben gekennzeichnete Beigresclumack nath längerer Aufhewahrung ebenfalls geltend macht. 
Bunsenbrenners etwa zur Hälfte vorsichtig abgeschmolzen. Diese so vorbereiteten Stückchen wurden theils direkt mit den verflüssigten Nährböden (Nährgelatine und Agar-Agarr) gemischt, theils in sterilem Wasser bei $27^{\circ}$ geschmolzen, kräftig mit letzterem geschüttelt und jedesmal $1 \mathrm{cem}$ des Wassers zur Untersuchung verwendet. Es gelang in keinem Falle, weder bei Zimmertemperatur, noch bei Brutschrankwärme auf den erwälınten Nährböden eine Entwicklung von Kolonien zu beobachten, sodass ich die von Zerner. nach welchen sich das PaImin stets als keimfrei erwies, nur bestätigen kann.

Dürften auf Grund dieser Feststellungen vom hygienischen Standpunkte keinerlei Einwendungen gegen die Beschaffenhcit und Verwendung des Palmins als menschliche Nahrung zu erheben sein, so ist die weitere Frage, ob nicht solche vom ernährungs-physiologischen Standpunkte geltend gemacht werden können.

Als ein günstiger Umstand in dieser Hinsicht ist das durch das Herstellungsverfahren bedingte fast gänzliche Fehlen von freien Fettsïuren zu erwähnen, deren Gebalt sich auch bei längerer Aufbewahrung, und zwar nur in den äussersten mit der Luft in Berülırung gewesenen Schichten, nur unwesentlich erhöht $\left.^{2}\right)$. Der ausserordentlich geringe Gehalt an fremden Beimengungen, insbesondere an Wasser und Eiweissstoffen, bedingt eine vorzügliche Haltbarkeit, indem dadurch die Neigung letztgenannter Stoffe, Anlass zur Bildung von Zersetzungen zu geben, auf ein Minimum herabgedrückt wird. 'Thatsachlich ist dem auch die Haltbarkeit und die geringe Neigung zum Ranzigwerden, wie bereits oben erwähnt, eine sehr grosse.

Da infolge der oben festgestellten Eigenschaften das Palmin in hygienischdiätetischer Hinsicht gewisse Vorzüge ${ }^{3}$ ) besitzt, würde es sich zunüchst darum handeln, festzustellen, ob und welche Erfahrungen bercits in dieser Hinsicht gesammelt sind, ob Palmin ebenso gut bekömmlich, ebenso leicht und vollkommen im Darm aufgenommen wird, als wir das von anderen Fetten, z. B. dem Butterfett, anzunehmen gewohnt sind. Stellen nun schon die besonderen Eigenschaften des Palmins (niedriger Schmelzpunkt, leichte Verseifbarkeit) letztere Annahme als sehr wahrscheinlich hin, so lassen dieselben andererseits jeden ungünstigen Einfluss auf die Verdauung von vornherein höchst zweifelhaft erscheinen. Die in dieser Richtung von Zerner angestellten künstlichen Verdauungsversuche mit einer Albuminlösung nach dem Verfahren von Kat ${ }^{4}$ ) haben ergeben, dass Palmin auf die Verdauung keinen irgendwie schädigenden Einfluss ausübt.

Bieten auch die beí der Krankenernährung mit Palmin gesammelten Erfahrungen, die in der bereits mehrfach crwähnten Abhandlung von Zerner

1) Leber Kokosbutter -- ein nenes Speisefett vou Th. Zeruer. Mittheilung ans der medicinischen Altheilung des Herrn Professor D rasche im k. k. allgemeinen krankenhause: za Wien; Centralblatt für die sesammte Therapie.

2) Sätregrad nach 8 Wochen in der äusseren schicht $=0,8$.

3) Vergl. Zerner.

4) Medicinische Blätter 1889, No. 27-29. 
niedergelegt sind, allerdings in erster Linie Interesse für den Arzt, so wird deren Kenntniss doch auch dem Nahrungsmittel-Chemiker insofern willkommen sein, als ihm bei event. Begutachtungen über den Gebrauchswerth des Palmins ete. IIinweise in der angedeuteten Richtung nur erwünscht sein können, weshalb ich die Jirgebnisse jener klinischen Beobachtungen im Folgenden kurz wiedergebe:

Die betr. Feststellungen erstreckten sich darauf, ob das Palmin gut und leicht von Gesunden und Kranken vertragen wird und ob es überhaupt der menschlichen Geschmacksrichtung entspricht. Zu diesem Zwecke wurden durch vier Wochen an 116 Kranke Speisen, und zwar mürbes Gebäck, Braten und Mehlspeisen, zu deren Darstellung als Ersatz der Fette ausschliesslich Kokosnussbutter verwendet wurde, verabreicht. Das Resultat der Beobachtungen wird dahin zusammengefasst, dass sich bei den Versuchen an Kranken die Kokosnussbutter als ein leicht verträgliches Fett, das auch bei Verdauungsstörungen keine Beschwerden verursacht, erwiesen hat. Unter den 116 Kranken, unter denen sich 8 akute und chronische Magenkatarrhe, 4 Magengeschwüre und Magenkrebse, 3 akute Darmkatarrhe, eine ganze Reihe von phthisischen und anämischen Dyspepsien befanden, hat keiner nach Verabreichung des mürben, mit Kokosnussbutter bereiteten Gebäckes - einer sonst wegen ihres Fettgehaltes nicht leicht zu vertragenden Speise iiber irgend eine Beschwerde geklagt, oder eine Verschlimmerung erfahren. Auch in drei Fällen, wo das Gebäck eine Stunde nach erfolgtem Erbrechen eingenommen wurde, wurden keine üblen F'olgen bemerkt, sondern im Gegentheil von den Kranken eine neue Gabe erbeten.

Besteht demgemäss kein Zweifel darüher, dass das Palmin als ein leicht verdanliches und für Gesunde und Kranke gleich gut bekömmliches Fett anzusehen ist, so erübrigte es noch, den Nährwerth des Präparates durch einige Versuche festzustellen. Da der Nährwerth in erster Linie von der mehr oder weniger vollständigen Verdaulichkeit abhängig ist, habe ich die nachstehend mitgetheilten beiden Ausnutzungsversuche zur Errmittelung des Verdaunngskoëfficienten angestellt.

Die als Nahrung dienende gemischte Kost, in welcher die einzelnen Xährstoffgruppen in normalen Verhältnissen zu einander standen, wurde unter möglichstem Ausschluss von Fremdfetten der Geschmacksrichtung, sowie die Versuche selbst den natürlichen Lebensverhältnissen der Versuchsperson ${ }^{1}$ ) angepasst. Die Dauer derselben betrug dreimal 24 Stunden; die verzehrten Mengen Palmin belicfen sich bei Periode I auf $136 \mathrm{~g}$, bei Periode II auf $90 \mathrm{~g}$ im Tage, liegen also innerhalb normaler Grenzen. Die Speisenordnung:) war

1) Zn den Versuchen wurde dieselbe Versuchsperson benutzt, welche bei ten früheren Perioden demsethen Zwecke gedient hatte. Tergl. diese Zeitselurift 1899, 2, 481 -506.

2) Früh $8: 2$ Chr lileine Tasse Kafee mit Zncker, aussertem 100 or Brot.

11 - a. $70 \mathrm{~g}$ Brot (event. Wein).

1' - Mitragulnot; nach dem kleine Tasse Kaffee nit Zucker.

$64:$ - abends $130 \mathrm{~g}$ Brot, kleine Tasse Theo und Bier. 
thiglich die gleiche. In Bezug auf sonstige Einzelheiten, betreffend Aufstellung der Nährstoff-Bilanz, analytische Bearbeitung des Untersuchungsmaterials und Berechnung der Versuchsergebnisse verweise ich auf die erste Veröffentlichung über "Margarine und Naturbutter" in dieser Zeitschrift (1899, 2, 481-506).

Die Versuchsergebnisse der einzelnen Tage sowie der Gesammt-Perioden waren folgende:

\section{Periode}

vom 24. bis 26. Januar 1899 .

1. Nährstoff-Bilanz des ersten Versuchstages.

\begin{tabular}{|c|c|c|c|}
\hline Einnabmen & Eiweiss & F'ett & Koblenhydrate \\
\hline $73 \mathrm{~g}$ Rindfleisch ${ }^{3}$ ) & $22, \tilde{\mathrm{o}} \mathrm{g}$ & $0,70 \mathrm{~g}$ & - \\
\hline 125 - Bohnen & $29,4-$ & $1,65=$ & $69,5 \mathrm{~g}$ \\
\hline $300-$ Brot & $18,7-$ & $0,31-$ & $149,3-$ \\
\hline 300 - Kartoffeln & $6,3-$ & $0,45-$ & $63,0-$ \\
\hline$\underline{20}$ - Tropon & $17,9-$ & 0,04 & - \\
\hline 15 - Mehl & $1, \bar{j}-$ & $0,15-$ & $11,3-$ \\
\hline 50 - Zucker & - & $\ldots$ & $49,5-$ \\
\hline 100 - Bier & $1,9-$ & 一 & $18,4-$ \\
\hline 136 - Palmin & - & $136,0-$ & - \\
\hline Im Gauzen & $98,2 \mathrm{~g}$ & $139,30 \mathrm{~g}$ & $361,0 \mathrm{~g}$ \\
\hline Verhältniss & 1 & 1,42 & 3,68 \\
\hline
\end{tabular}

Nährstoff-Verhäiltniss: $1: 7,22$.

Gehalt an Fremdfetten: $2,4 \%$.

Kothmenge am 2. Tage: 27,35 g lufttr. Substanz mit 92,91\% $=25,40 \mathrm{~g}$. Trockensubst. Darin Fett $\left.^{5}\right): 2,73 \mathrm{~g}$; also Fett verdant: 98,04 ${ }^{\circ}, 0$ -

2. Nährstoff-Bilanz des zweiten Versuehstages.

\begin{tabular}{|c|c|c|c|}
\hline Einuahmen & Eiweiss & Fett & Koblenbydrate \\
\hline $73 \mathrm{~g}$ Rindfleisch & $22, \bar{g} \mathrm{~g}$ & $0,70 g$ & -- \\
\hline 125 - Bohnen & $29,4-$ & $1,65-$ & $69,5 \mathrm{~g}$ \\
\hline 305 - Brot & $19,1-$ & $0,33-$ & $152,0-$ \\
\hline 300 - Kartoffeln & $6,3-$ & $0,40-$ & $63,0-$ \\
\hline 20 - 'Tropon & $17,9-$ & $0,04-$ & $\ldots$ \\
\hline $1 \overline{5}-\mathrm{Mehl}$ & $1,5-$ & $0,1.5-$ & $11,3-$ \\
\hline Ј0 - Zucker & -- & $'-$ & $+9,5-$ \\
\hline 400 - Bier & $1,9=$ & - & $18,1-$ \\
\hline 136 - Palmin & - & $1: 36,00-$ & - \\
\hline $\begin{array}{l}\text { Im Ganzen } \\
\text { Verhältniss }\end{array}$ & $98,6 \mathrm{~g}$ & $139,32 \mathrm{~g}$ & $\begin{array}{c}363,7 \mathrm{~g} \\
3,69\end{array}$ \\
\hline
\end{tabular}

Nährstoff-Verhältniss: $1: 7,22$.

Gehalt an Fremdfetten: $2,4 \%$.

Kothmenge am 3. Tage: $37,00 \mathrm{~g}$ lufttr. Substanz mit 93,70\% $=34,67 \mathrm{~g}$. Trockensubst. Darin Fett' ${ }^{\prime}$ : 4,43 $\mathrm{g}$; also Fett verdant: 96, $\$ 2 \%$.

1) Gekochtes Rindfleisch nit $0,96 \%$ Fett und $30,8 \%$ Fiweiss.

2) Bohnen mit $1,53 \%$ Fett.

3) $5 \mathrm{~g}$ lufttr. Sulstanz $=0,5000 \mathrm{~g}$ A Aetherextrakt $=10,00\} 9,970_{0}^{\circ}$

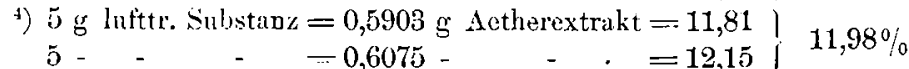


3. Nährstoff-Bilanz des dritten Versuchstages.

\begin{tabular}{|c|c|c|c|}
\hline Einnahmen & Eiweiss & Fett & Kohlenhydrate \\
\hline $73 \mathrm{~g}$ Rindfleisch & $29, \bar{\prime}$ & $0,70 \mathrm{~g}$ & - \\
\hline 125 - Bohnen & $29,4-$ & $1,65=$ & $69,5 \cong$ \\
\hline $300-$ Brot & $18,8-$ & $0,33-$ & $149,1-$ \\
\hline $300-$ Kartoffeln & $6, ; 3$ & 0,45 & $63,0-$ \\
\hline 20 .. Tropon & $17,9-$ & $0,0.4-$ & - \\
\hline $15-$ Mehl & $1, \overline{;}-$ & $0,15-$ & 11,3 \\
\hline 50 - Zucker & - & -- & $49,5-$ \\
\hline 400 - Bier & $1,9-$ & 一 & $18,4-$ \\
\hline 136 - Palmin & - & $136,00-$ & - \\
\hline $\begin{array}{l}\text { Im Ganzen } \\
\text { Verhältniss }\end{array}$ & $\begin{array}{c}98,3 g \\
1\end{array}$ & $\begin{array}{c}139,32 \text { o } \\
1,42\end{array}$ & $\begin{array}{c}361,18 \\
3,67\end{array}$ \\
\hline
\end{tabular}

Nährstoff-Verhältniss: $1: 7,22$.

Gehalt an Fremdfetten: $2,4 \%$.

Kothmenge am 4. Tage: $40,73 \mathrm{~g}$ lufttr. Substanz mit $94,26^{\circ} \%=38,39 \mathrm{~g}$ Trockensubst. Darin Fett $^{1}$ ): 4,07 $g$; also Fett verdaut: $\mathbf{9 7 , 0 8} \%$.

Zasammenstellung der Versuchsergebnisse der I. Periode.

\begin{tabular}{|c|c|c|c|c|c|c|}
\hline \multirow{2}{*}{$\begin{array}{c}\text { Bezeichnung } \\
\text { der } \\
\text { Tage }\end{array}$} & \multirow{2}{*}{$\begin{array}{c}\text { Einnahmen } \\
\text { Gesammtfett } \\
\mathrm{g} \\
\end{array}$} & \multicolumn{4}{|c|}{ Ausgaben } & \multirow[b]{2}{*}{$\begin{array}{c}\text { Fett aufge- } \\
\text { nommen } \\
0 / n \\
\end{array}$} \\
\hline & & $\begin{array}{c}\text { Koth, } \\
\text { lufttrocken } \\
\alpha \\
\end{array}$ & $\begin{array}{c}\begin{array}{c}\text { Koth.Trocken } \\
\text { substanz } \\
g\end{array} \\
\end{array}$ & $\begin{array}{c}\text { Fett in der } \\
\text { Koth-Trocken- } \\
\text { substanz } \\
0 / n\end{array}$ & $\begin{array}{c}\text { Gesammt-Fett } \\
\underline{\underline{p}}\end{array}$ & \\
\hline 1. Tag. & 139,30 & 27,35 & 25,40 & 10,73 & 2,73 & 98,04 \\
\hline $2 . \quad-$ & 139,32 & 37,00 & 34,67 & 12,79 & 4,43 & 96,82 \\
\hline 3. - & 139,32 & 40,73 & 38,39 & 10,62 & 4,07 & 97,08 \\
\hline m Ganzen & 417,94 & 105,08 & $98, \mathbf{4 6}$ & - & 11,23 & - \\
\hline In 24 Stuuden & 139,31 & 35,03 & 32,82 & 11,38 & 3,74 & 97,31 \\
\hline
\end{tabular}

\section{Periode}

rom 1. lis 3. Felmuar: 1899.

1. Nährstoff-Bilanz des ersten Versuchstages.

\begin{tabular}{|c|c|c|c|}
\hline Einnalumen & Eiweiss & Fett & Kohleuhydrate \\
\hline $120 \mathrm{~g}$ Rindfleisch ${ }^{2}$ ) & $39,6 \mathrm{~g}$ & $2,70 \mathrm{~g}$ & - \\
\hline 130 - Bohnen & $30,6-$ & $1,72-$ & $72,3 g$ \\
\hline 300 - Brot & $18,7-$ & 0,32 & $149,-1-$ \\
\hline 300 - Kartoffeln & $6,3-$ & 0,45 & $63,0-$ \\
\hline 20 - Tropon & $17,9-$ & $0,04-$ & - \\
\hline 50 - Zucker & & - & $49,5-$ \\
\hline $100-$ Wein & - & - - & - \\
\hline 400 - Bier & $1,9-$ & - & $18, \pm-$ \\
\hline 90 - Palmin & - & $90,00-$ & - \\
\hline $\begin{array}{l}\text { Tin Ganzen } \\
\text { Terhältniss }\end{array}$ & $\begin{array}{c}115,0 \mathrm{~g} \\
1\end{array}$ & $\begin{array}{c}9.5,238 \\
0,83\end{array}$ & $\begin{array}{c}352,6 \mathrm{~g} \\
3,07\end{array}$ \\
\hline
\end{tabular}

1) $5 \mathrm{~g}$ lufttr. Substanz $=0,5213 \mathrm{~g}$ Aetherextrakt $=10,43 \quad 10,01 \%$

2) Gekochtes Rinlffeisch mit $2,25 \%$. Fett und $33,0^{\prime \prime}{ }_{0}^{\prime}$ Eiweiss. 
Nährstoff-Verhăltnis : $1: \overline{0}, 14$.

Gehalt an Fremdfetten: $\overline{0}, 5 \%$ :

Kothmenge am 2. Tage: $41,42 \mathrm{~g}$ lufttr. Substanz mit 92,61\% $=38,36 \mathrm{~g}$ Trockensubst. Darin Fett $^{1}$ ): $5,35 \mathrm{~g}$; also Fett verdant: $94,38 \%$.

2. Nährstoff-Bilanz des zweiten Versuchstages.

\begin{tabular}{|c|c|c|c|}
\hline Einnabmen & Eiweiss & Fett & Koblenhydrate \\
\hline $120 \mathrm{~g} \cdot$ Rindfleisch & $39,6 \mathrm{~g}$ & $2,70 \mathrm{~g}$ & - \\
\hline 130 - Bohnen & $30,6-$ & $1,72-$ & $72,3 \mathrm{~g}$ \\
\hline $300-$ Brot & $18,7-$ & $0,32-$ & $149,4-$ \\
\hline 300 - Kartoffeln & $6,3-$ & $0,45-$ & $63,0-$ \\
\hline $20-$ Tropon & $17,9-$ & $0,04-$ & -- \\
\hline Б̃o - Zucker & - & .. - & $49,5-$ \\
\hline $100-$ Wein & - & - & $\cdot$ \\
\hline $400-$ Bier & $1,9-$ & - & $18,1-$ \\
\hline 90 - Palmin & - & $90,00-$ & - \\
\hline Im Ganzen & $115,0 \mathrm{~g}$ & $95,23 g$ & $352,6 \mathrm{~g}$ \\
\hline Verhältniss & 1 & 0,83 & 3,07 \\
\hline
\end{tabular}

Nährstoff-Verhältniss: $1: \check{0}, 14$.

Gehalt an Fremdfetten: $5,5 \%$.

Kothmenge am 3. Tage: $42,70 \mathrm{~g}$ lufttr. Substanz mit $92,32 \%=39,42 \mathrm{~g}$ Trockensubst. Darin Fett $\left.{ }^{2}\right): 4,58 \mathrm{~g}$; also Fett verdunt: $95, \mathbf{1 9}^{\circ}{ }_{0}$.

3. Nährstoff-Bilanz des dritten Versuchstages.

\begin{tabular}{|c|c|c|c|}
\hline Einnahmen & Hineiss & F'eft & Koblenhydrate \\
\hline $120 \mathrm{~g}$ Rindfleisch & $39,6 \mathrm{~g}$ & $2,70 \mathrm{~g}$ & - \\
\hline 130 - Bohnen & $30,6-$ & 1,72 & $72,3 \mathrm{~g}$ \\
\hline $300-$ Brot & $18,7-$ & $0,32-$ & $149,4-$ \\
\hline 300 - Kartoffeln & $6,3-$ & $0,45-$ & $63,0-$ \\
\hline 20 - Tropon & $17,9-$ & 0,04 & - \\
\hline 50 - 7ucker & -- & - & $49,5-$ \\
\hline $100-$ Wein & - & $\cdots$ & - \\
\hline 400 - Bier & $1,9-$ & - & $18,4-$ \\
\hline $90-$ Palmin & $\ldots$ & 90,00 - & - \\
\hline Im Ganzen & $115,0 \mathrm{~g}$ & $95,23 \mathrm{~g}$ & $352,6 \mathrm{~g}$ \\
\hline Verhältniss & 1 & 0,83 & 3,07 \\
\hline
\end{tabular}

Nährstoff-Verhältniss: $1: 5,14$.

Gehalt an Fremdfetten: 5,5\%.

Kothmenge am 4. Tage: $30,80 \mathrm{~g}$ lufttr. Substanz mit $93,790_{0}^{\prime}=28,89 \mathrm{~g}$. Trockensubst. Darin Fett $^{3}$ ) 2,91 $\mathrm{g}$; also Fett verdant: 96,94\%.

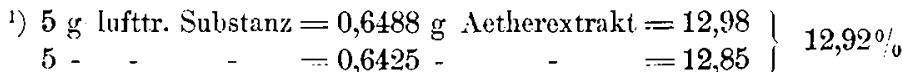

$$
\begin{aligned}
& \text { 2) } 5 \mathrm{~g} \text { lufttr. Substanz }=0,5375 \mathrm{~g} \text { Letherextrakt }=10,75 \\
& 5 \text { - - - }=0,5347-\quad-\quad=10,69\} 10,72_{0}^{\circ} \\
& 5 \text { - - - } \quad-0,5365 \text { - } \quad=10,73
\end{aligned}
$$

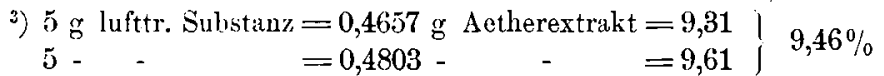


Znsammenstellung der Versuchsergebnisse der II. Periode.

\begin{tabular}{|c|c|c|c|c|c|c|}
\hline \multirow[b]{2}{*}{$\begin{array}{c}\text { Bezeichnung } \\
\text { der Tage }\end{array}$} & \multirow{2}{*}{$\begin{array}{c}\text { Einnahmen } \\
\text { Gesammt-Fett } \\
q\end{array}$} & \multicolumn{4}{|c|}{ Ausgaben } & \multirow{2}{*}{$\begin{array}{c}\text { Fett } \\
\text { anfge- } \\
\text { nommen } \\
\% \\
\end{array}$} \\
\hline & & $\begin{array}{c}\text { Koth } \\
\text { lufttrock en } \\
\mathrm{g} \\
\end{array}$ & $\begin{array}{c}\text { Koth-Trocken- } \\
\text { substanz } \\
\mathrm{g}\end{array}$ & $\begin{array}{c}\text { Fett in der } \\
\text { Koth-Trocken } \\
\text { substanz } \\
0 / n \\
\end{array}$ & $\begin{array}{c}\text { Gesammt-F ett } \\
g \\
\end{array}$ & \\
\hline 1. Tag & 95,23 & 41,42 & 38,36 & $13,9 \check{5}$ & 5,35 & 94,38 \\
\hline & 95,23 & 42,70 & 39,42 & 11,61 & 4,58 & 95,19 \\
\hline 3. & 95,23 & 30,80 & 28,89 & 10,09 & 2,91 & 96,94 \\
\hline $\begin{array}{l}\text { Im Ganzen } \\
\text { in } 24 \text { Stunden }\end{array}$ & $\begin{array}{r}285,69 \\
95,23\end{array}$ & $\begin{array}{r}\mathbf{1 1 4 , 9 2} \\
\mathbf{3 8 , 3 1}\end{array}$ & $\begin{array}{r}106,67 \\
\mathbf{3 5 , 5 6}\end{array}$ & $\overline{11,88}$ & $\begin{array}{r}12,84 \\
\mathbf{4 , 2 8}\end{array}$ & 95,50 \\
\hline
\end{tabular}

Die Verdauung während der beiden Perioden verlief ohne Störungen und es ergiebt sich auf Grund der vorstehenden Ansnutzungsversuche im Mittel eine Verdaulichkeit von rund $96,4 \%$. Die absolute Verdaulichkeit würde, verglichen mit den für Butter und Margarine ermittelten Werthen (96,96\% bezw. 96,27\%), folglich als in der Mitte zwischen beiden liegend anzunehmen sein.

Der Unterschied der Versuchsergebnisse beider Perioden beträgt $1,8 \%$, ist also nicht ganz unerheblich. Forschen wir näher nach der Ursache dieser Differenz, so kann dieselbe durch verschiedene Umstände bedingt sein. Wie ersichtlich, sind die Versuche weder unter gleichen Bedingungen noch mit denselben Mengen Palmin angestellt worden. Bei im Allgemeinen gleichartigen Speisen sind deren Mengenverhältnisse bei den beiden Perioden so versehieden gestaltet worden, dass sich im einen Falle eine Kost mit engem und im anderen Falle eine solche mit weiterem Nährstoffverhältnisse ergab, um gleichzeitig festzustellen, ob und welchen Einfluss die veränderte Zusammensetzung der Nahrung auf die Verdaulichkeit der Fettsubstanz ausüben würde. Um die beiden Resultate mit einander vergleichbar zu machen, war es von Wichtigkeit, dass die mit der Nahrung verzehrten Fremdfette, deren absolute Verdaulichkeit noch keineswegs als völlig feststehend anzunehmen ist, ihrer Menge nach entweder so gering blieben, dass sie die Resultate nicht oder nur unmerklich beeinflussen konnten, oder da dies bei Verwendung der natürlichen Nahrungsmittel nicht der Fall ist, dass sowohl die absolute Menge der Fremdfette, als auch deren einzelne, je nach ihrer Herkunft verschiedenen Bestandtheile, in beiden Fällen wenigstens annähernd die gleichen waren. Bezüglich der pflanzlichen Nahrungsmittel ist dieser Forderung genau zu entsprechen. Schwieriger dagegen gestaltet sich die Sache, wenn man, wie im vorliegenden Falle bei gemischter Kost, nicht gänzlich auf Fleischnahrung verzichten will. Für die kurze Dauer einer Periode ist es ja möglich, eine Probe mit gleichmässigem Fettgehalt herzustellen; bei der geringen Haltbarkeit des Fleisches dürfte es jedoch kaum angängig sein, eine entsprechende Theilprobe, ohne Zuflucht zur Konservirung') in Kühlräumen, die niclıt überall zur Verfügung stehen, nehmen zu müssen, für eine zweite, obendrein noch zeitlich von

1) Chemische Konservirungsmittel kommen schon ans dem Grunde nicht in Betracht weil deren Wirkung lei dem Verdaungsrorgange unberechenbar ist. 
der ersten getrennte Periode genussfähig zu erhalten. Man wird unter den gegebenen Umständen sich damit begnügen müssen, bei der Auswahl eines fettarmen Fleisches möglichst sorgsam zu verfahren. Trotzdem ich diesen Weg bei der II. Periode eingeschlagen habe, ist es mir nicht gelungen, den gewünschten Verhältnissen Rechnung zu tragen, insofern als die Fettsubstanz des gekochten Rindfleisches, wie die spätere Analyse ergab, doch noch $2,25 \%$ betrug. Infolge dessen steigt die aus der Nahrung stammende absolute Menge an Fremdfetten von $3,3 \mathrm{~g}$ bei Periode I auf ca. $5,2 \mathrm{~g}$ im Tage bei Periode II.

Bei der näheren Betrachtung der vorstehend mitgetheilten Ergebnisse erscheint es auf den ersten Blick nicht recht verständlich, dass in einem Falle bei einer Gabe von nur $90 \mathrm{~g}$ Palmin im Tage 95,5\%, im anderen Falle bei der $11 / 2$-fachen Gabe von $136 \mathrm{~g}$ dagegen $97,3 \%$, also rund 1,8\% mehr Fettsubstanz verdaut worden sind. Will man nicht annehmen, dass dieser Untersehied in der Verdaulichkeit vielleicht in Folge der Veränderung des Nährstoff-Verhältnisses der Nahrung hervorgerufen sein könnte, so wird man geneigt sein, sie in erster Linie einer nur geringen Aufnahme der Fremdfette zuzuschreiben. Die Richtigkeit dieser Annahme vorausgesetzt, werden die Ergebnisse sofort verständlicher erscheinen, denn je grösser dic Menge der verzehrten Fremdfette ist, einen um so grösseren Rückstand (Aetherextrakt) werden sie hinterlassen. Die Erhöhung der Fremdfette von $3,3 \mathrm{~g}$ bei Periode I auf 5,2 $\mathrm{g}$ im Tage bei Periode II, welche lediglich durch die Fettsubstanz des Rindfleisches hervorgerufen ist, hat eine Vermehrung des Aetherextraktes von $3,74 \mathrm{~g}$ bei Periode I auf $4,28 \mathrm{~g}$ im Tage bei Periode II zur Folge gehabt, trotzdem im letzteren Falle $46 \mathrm{~g}$ Palmin weniger verzehrt wurden. Vergleicht man diesen Befund mit früheren, bei den Versuchen über die Verdaulichkeit von Margarine und Naturbutter $^{1}$ ) gemachten Beobachtungen, so ergeben sich dort ganz ähnliche Verhältnisse. Ich führe dieselben der Vollständigkeit halber nachstehend an:

Bei Periode I betrug der Gebalt an Fremdfetten während der ersten Hälfte im Tage $3,11 \mathrm{~g}$ (davon $0,93 \mathrm{~g}$ aus Rindfleisch stammend), die verzehrte Menge an Margarinefett 141,6 $\mathrm{g}$ und der Aetherextrakt des Darmkothes im Tage 3,83 $\mathrm{g}$. Bei dẹ erhöhten Gabe an Rindfleisch während der zweiten Hälfte der Periode betrug der Gehalt an Fremdfetten $3,73 \mathrm{~g}$, wovon alleiu 2,77 $\mathrm{g}$ Rindstalg waren; trotzdem nur $133 \mathrm{~g}$ Margarinefett verzehrt wurden, stieg der $\Lambda$ etherextrakt auf $5,42 \mathrm{~g}$ im Tage, die Erhöhung betrug also rund $1,6 \mathrm{~g}$.

Bei Periode IV zeigt sich dieselbe Erscheinung.

$\begin{array}{cccc}\text { Fremdfette } & \begin{array}{c}\text { Verzehrtes } \\ \text { Butterfett }\end{array} & \begin{array}{c}\text { Aetherextrakt } \\ \text { des Darrakothes }\end{array} \\ \text { I. Hälfte } & 4,44 \mathrm{~g} \text { (davon } 2,74 \text { Rindstalg) } & 107,04 \mathrm{~g} & \mathbf{4 , 4 4} \mathrm{g} \\ \text { II. Hälfte } & 5,06-(-3,43- & 107,04- & 5,19-\end{array}$

Lassen diese unter den verschiedensten Verhältnissen ermittelten Werthe einen Schluss $z u$, so lässt sich mit Bestimmtheit nur sagen, dass, sobald die in dem verzehrten Rindfleisch befindliche absolute Menge an Fettsubstanz grösser

1) Vergl. die Arbeit über die Verdaulichlkeit von Margarine und Naturbutter in dieser Zeitschrift 1899, 2, 484-506. 
wurde, in allen Fällen auch eine Vermehrung des Aetherauszuges des Darmkothes stattgefunden hat.

$\mathrm{Ob}$ und in welchem Maasse sich das zwischen den Muskelfasern abgelagerte Rindsfett im vorliegenden Falle an der Vermehrung der Aetherauszüge betheiligt hat, lässt sich ohne eingehende Untersuchungen nicht feststellen. Jedenfalls bildet die einfache Thatsache, auf diese Versuche übertragen, eine ungezwungene Erklärung für die Erhöhung des Aetherextraktes bei Periode II und mithin auch für die Erniedrigung des aus demselben durch Rechnung gefundenen Verdauungskoëfficienten. Ceber die procentige Zusammensetzung des Aetherextraktes der beiden Perioden giebt nachstehende Tabelle Aufschluss.

\begin{tabular}{|c|c|c|c|c|c|c|c|c|c|}
\hline \multirow[b]{2}{*}{ Periode } & \multicolumn{3}{|c|}{ Unverseifbare Substanz } & \multicolumn{5}{|c|}{ Verseifbare Substanz (Rückstand C.) } & \multirow[b]{2}{*}{$\begin{array}{c}\text { Leci- } \\
\text { thinge- } \\
\text { halt } \\
\% \\
\%\end{array}$} \\
\hline & \begin{tabular}{|c|} 
Rück- \\
stand A, ent- \\
standen bei \\
der Versei- \\
fung mit \\
alk. Kali \\
$\% \%$ \\
\end{tabular} & \begin{tabular}{|c|} 
Rückstand B \\
aus der \\
Seifenlösung \\
mit Petrol- \\
äther auf- \\
genommen \\
$\%$ \\
\end{tabular} & $\begin{array}{c}\text { Schmelzpunkt } \\
\text { von B } \\
{ }^{\circ} \mathrm{C}\end{array}$ & $\begin{array}{c}\text { Ge- } \\
\text { sammt- } \\
\text { Fett } \\
\text { als Fett- } \\
\text { säuren } \\
\% \\
\% \\
\end{array}$ & $\begin{array}{l}\text { Sehmelzpunkt } \\
{ }^{\circ} \mathrm{C}\end{array}$ & $\begin{array}{l}\text { Jod- } \\
\text { zahl }\end{array}$ & $\begin{array}{l}\text { Yersei- } \\
\text { fungs: } \\
\text { zahl }\end{array}$ & $\begin{array}{c}\text { Refrak- } \\
\text { tion } \\
\text { bei } 50^{0}\end{array}$ & \\
\hline $\mathrm{I}$ & 12,2 & 3,8 & $\begin{array}{c}\text { wird allmählich } \\
\text { von } 40^{\circ} \text { an weich; } \\
\text { Hauptmasse } \\
\text { schmilzt } \\
\text { zwischen } 72 \\
\text { und } 73 \text { ? }\end{array}$ & 78,1 & $\begin{array}{c}\text { weich bei } 29^{\circ} ; \\
\text { Hauptmasse bei } \\
32,5-33,5 ; \text { völlig } \\
\text { geschmolzen } \\
\text { bei } 35^{0}\end{array}$ & 16,6 & 124,8 & 72,3 & 一 \\
\hline II & 13,5 & $3,3]$ & $\begin{array}{c}\text { weich bei } 40^{\circ} \\
\text { Hauptmasse } \\
\text { schmilzt bei } 70^{\circ} \\
\text { und ist bei } 73^{\circ} \\
\text { völlig ge- } \\
\text { sehmolzen }\end{array}$ & 80,0 & $\begin{array}{c}\text { weich bei } 30^{\circ} ; \\
\text { Hauptmaase } \\
\text { zwischen } 32-35 \text {; } \\
\text { völlig geschmol- } \\
\text { zen bei } 36^{\circ}\end{array}$ & 19,9 & 94,7 & 78,0 & 12,7 \\
\hline
\end{tabular}

Die Rückstände A und B zeigen dieselben specifischen Eigenschaften, wie solche bei den entsprechenden Verbindungen früher beschrieben ${ }^{1}$ ) sind; auffallend ist, dass der krystallinisch erstarrende, gelbroth gefärbte Rückstand $\mathbf{B}$ im vorliegenden Falle mit nur ca. 3,5 \% gegen ca. 33,0 bei den bereits mehrfach erwähnten früheren Versuchen betheiligt ist. Demgemäss beträgt denn auch der Gehalt an "Fett" in diesem Falle rund $80 \%$ gegen nur $50 \%$ früher. Irgend welche positiven Schlüsse in Bezug auf Zusammensetzung und Herkunft der Fettsäuren lassen sich aus den analytischen Werthen nicht ziehen; in negativem Sinne lässt sich nur das sagen, dass kein Anhalt gegeben ist, die verseifbare Substanz als unverändertes bezw. unverdautes Palmin anzusehen.

Es ergiebt sich aus dem Vorstehenden, dass die absolute Verdaulichkeit cles Palmins nicht hinter derjenigen anderer Nahrungsfette zurücksteht, und wer mit mir zu der Ansicht neigt, dass das im Aetherextrakte vorbandene wirkliche „Fett" grösstentheils Stoffwechselprodukten seine Entstehung verdankt und vielleicht nur zum geringsten Theil einer unvollständigen Aufnahme der verzehrten Fettsubstanzen zuzuschreiben ist, wird keine Einwendungen dagegen erheben, wenn man die Verdaulichkeit des Palmins ebenso wie die der Butter und Margarine als eine absolute annimmt.

1) Vergl. diese Zeitschrift 1899, 2, 502. 